\title{
Late return of diaphragm function in a ventilator-dependent patient with a high cervical tetraplegia: case report, and interactive review
}

\author{
William O McKinley \\ Department of Physical Medicine and Rehabilitation, Medical College College of Virginia, Richmond, Virginia, USA
}

\begin{abstract}
Return of diaphragm function in an individual with ventilator-dependent high cervical tetraplegia is presented. The patient was maintained on a ventilator for five years after sustaining a C3-C4 vertebral fracture with a complete high cervical tetraplegia. Diaphragmatic fluoroscopic evaluation at the initial injury time revealed a nonfunctioning diaphragm, and the patient was subsequently discharged home on a portable ventilator. Respiratory evaluation 5 years post injury, including, diaphragmatic fluoroscopy, transcutaneous phrenic nerve conduction studies and pulmonary function tests, revealed intact phrenic nerve function bilaterally with spontaneous diaphragmatic motion. Subsequently, successful ventilatory weaning and diaphragmatic muscle strengthening was achieved. This case report emphasises the importance of serial evaluation of phrenic nerve viability, diaphragmatic function and ventilator dependency in patients with a high cervical tetraplegia in light of potential neurological recovery.
\end{abstract}

Keywords: tetraplegia; diaphragm function; phrenic nerve; mechanical ventilation; respiratory weaning

Ventilatory assistance in high cervical spinal cord injury (SCI) becomes necessary when there is interruption of the normal neurological control of respiration resulting in hypoventilation. Following spinal cord injury at the C4 neurological level or above (high cervical tetraplegia), supraspinal control of the diaphragm is lost and the respiratory muscles below the level of the injury become paralyzed. ${ }^{1}$ This impairs the injured patient's ability to expand the lungs and chest wall effectively and assisted ventilation is required for adequate respiration. ${ }^{2} \mathrm{~A}$ review of the literature reveals that as many as $4 \%$ of patients admitted to major spinal cord injury centers have sustained a SCI at the $\mathrm{C} 4$ neurological level or above, and require respiratory assistance. ${ }^{3}$ Potential complications associated with prolonged assisted ventilation include increased patient morbidity and cost, and diminished quality of life. ${ }^{4,5}$ The number of individuals surviving with complete or incomplete high tetraplegia is increasing, ${ }^{6}$ in part, secondary to improved early management and resuscitative efforts. Furthermore, it has been shown that individuals with a complete or incomplete SCI have potential for recovery of neurological function within the zone of injury, ${ }^{7}$ which in ventilator-dependent high cervical tetraplegia, may lead to recovery of diaphragm function and improved voluntary respiratory ability.

Studies have reported increased long-term survival with ventilatory-independent tetraplegia 4 and vigorous efforts need to be undertaken to wean individuals from mechanical ventilation. The importance of re-evaluation of respiratory function and phrenic nerve viability in such patients is stressed in this clinical report. Reevaluation, utilizing physical examination, diaphragmatic fluoroscopy, phrenic nerve conduction studies, and pulmonary function tests are required to appropriately assess respiratory status before (and after) active respiratory exercise training can be undertaken.

\section{Case report}

A 23-year old male patient with tetraplegia was admitted to a tertiary university spinal cord injury rehabilitation unit for evaluation of his respiratory status and ventilator dependency. He had sustained a high cervical tetraplegia five years prior to admission as a result of a motor vehicle accident which resulted in immediate loss of sensation and active movement below the neck, and he required resuscitative efforts and immediate ventilatory assistance. Neurological examination at the time of his initial injury revealed C3 complete tetraplegia with loss of all sensation in the C4 dermatome and below, and with no spontaneous respiratory ability. X-rays revealed a C3-C4 vertebral fracture, and diaphragmatic fluoroscopic examination 8 days after his initial admission showed that there was no active movement of either hemidiaphragm. He was hospitalized for approximately 8 months following the initial injury, and repeated attempts to wean mechanical ventilation were unsuccessful secondary to poor vital capacities and inspiratory pressures. He returned home with his family dependent on a portable 
ventilator for 24 h-a-day ventilatory assistance. During the past 5 years he had required readmission on numerous occasions secondary to respiratory complications. None of these readmissions were to our facility and there were no indications of follow-up diaphragmatic evaluation with electrodiagnosis, fluoroscopy, or pulmonary function studies.

Five years following his initial injury, he presented to our SCI outpatient clinic for routine clinical followup. He had experienced several instances when for various intervals ('several minutes') he would be disconnected from the ventilator with only mild dyspnea. The patient was scheduled for admission to our SCI Rehabilitation Unit for reevaluation of his respiratory status and ventilator dependency. Physical examination on this admission revealed a young male patient, cooperative and in good spirits. His breathing was maintained by a portable ventilator, attached through a tracheostomy. Parameters were set at a tidal volume of $1200 \mathrm{cc}, 12$ breaths/minute $(\mathrm{bpm})$ and $\mathrm{FIO}_{2}$ of 0.8 . Neurological examination revealed complete $\mathrm{C} 4$ tetraplegia with loss of all sensation in the $\mathrm{C} 5$ dermatome and below, including the sacral segments. Motor examination revealed good strength in neck musculature and shoulder elevation but loss of all other active movement in the upper and lower extremities. There appeared to be no active chest or abdominal rise during voluntary inspiratory effort. Reflexes in all of the extremities were hyperactive.

Respiratory re-evaluation was begun at this time and included chest X-ray, arterial blood gases, diaphragmatic fluoroscopy, phrenic nerve conduction studies and pulmonary function studies. The chest roentgenogram was normal. Pulmonary function studies revealed a vital capacity of $500 \mathrm{cc}$ 's. Arterial blood gases were observed both with the patient on the ventilator $\left(\mathrm{pH} 7.44, \mathrm{SaO}_{2} 97 \%, \mathrm{PaO}_{2} 90, \mathrm{PaCO}_{2}\right.$ 40) and with ventilator support discontinued for up to $10 \min \left(\mathrm{pH} 7.40, \mathrm{SaO}_{2} 94 \%, \mathrm{PaO}_{2} 84, \mathrm{PaCO}_{2} 44\right)$. No spontaneous chest wall or abdominal rise was noted though accessory neck musculature was active with a rate of $20 \mathrm{bpm}$. Fluoroscopy of the diaphragm without ventilator assistance revealed bilateral diaphragm motion of approximately $2-3 \mathrm{~cm}$. Bilateral percutaneous phrenic nerve conduction studies (NCS) were performed and revealed a motor latency of $7.9 \mathrm{msec}$ and amplitude of $450 \mathrm{uv}$ on the left and a motor latency of $13.5 \mathrm{msec}$ and amplitude of $212 \mathrm{uv}$ on the right. In our laboratory, this was consistent with normal NCS's on the left and mildly delayed motor latency/reduced amplitude on the right. Fluoroscopy of the diaphragm during phrenic nerve stimulation revealed a $6-8 \mathrm{~cm}$ movement of the diaphragm bilaterally.

Based on this evaluation, a weaning program was slowly and deliberately undertaken utilizing progressive ventilatory-free time on a T-tube over a period of 7 days while monitoring inspiratory and expiratory pressures, vital capacity, sequential arterial blood gases and clinical examination. The patient was maintained ventilator-free on intermittent 2 liter nasal canula and diaphragmatic resistive strengthening exercises were continued using low-weight abdominal pads. Pulmonary function testing independent of ventilator assistance showed a vital capacity of 870 cc's. Repeat diaphragmatic fluoroscopy following ventilatory weaning was consistent with diaphragmatic movement of $4-6 \mathrm{~cm}$ bilaterally. No further complications were noted and he was discharged home 10 days after extubation. Follow-up at 1 year post mechanical ventilatory-weaning revealed that the patient had remained totally independent of assisted ventilation, free of respiratory complications and his tracheostomy tube had been removed.

\section{Discussion}

Literature reveals that as many as $4 \%$ of patients admitted to major spinal cord injury centers have a $\mathrm{SCI}$ at the $\mathrm{C} 4$ neurological level or above and require respiratory assistance. ${ }^{3}$ The number of individuals surviving with high tetraplegia and/or incomplete tetraplegia is increasing, ${ }^{6}$ in part, secondary to improved early management and resuscitative efforts. Furthermore, it has been shown that individuals with a complete (defined as absent sacral sensory and motor function) and an incomplete SCI have potential for recovery of neurological function within the zone of injury. $^{7}$ In ventilator-independent high cervical tetraplegia this may lead to recovery of diaphragm function and improved voluntary respiratory ability.

We have presented a patient with a high cervical tetraplegia whose initial voluntary diaphragm function was absent, only to become functional at a later date. Ventilatory assistance in those with a high cervical spinal cord injury becomes necessary when there is interruption of the normal neorological control of respiration resulting in hypoventilation. ${ }^{2}$ The diaphragm is innervated by the phrenic nerve (with contributions from cervical cord segments 3, 4 and 5 ), and is considered to contribute nearly $65 \%$ of the vital capacity of the lung. ' Following SCI at the C4 neurological level or above, supraspinal control of the diaphragm is lost and the respiratory muscles below the level of injury become paralyzed. This leaves the individual with only some of the accessory muscle of respiration, mainly the sternocleidomastoid and trapezius. Accessory muscles play a minimal role in normal respiration, and mechanical ventilatory assistance is most always required for survival after such an injury. ${ }^{8}$ There are several alternative respiratory support measures available for ventilator-dependent individuals $^{9-11}$ including: body ventilators, noninvasive intermittent positive airway pressure (delivered via mouth, nose or both), phrenic nerve stimulation and glossopharyngeal breathing.

Cervical spinal cord injury leads to compromised respiratory function including reduced lung volumes, vital capacities and decreased ability to cough. ${ }^{12}$ Some 
patients with a high cervical injury but intact diaphragmatic function require artificial ventilation as a temporary measure. ${ }^{4,9}$ Investigators of acute cervical SCI subjects have found an initial moderate decrease in vital capacity. McMichan followed 22 tetraplegic patients and found that the vital capacity volumes averaged $30 \%$ of normal immediately following injury and rose to $54 \%$ of normal at 5 months. ${ }^{3} \mathrm{He}$ attributed improvements to both diaphragmatic strengthening combined with increased stability of the rib cage and abdominal wall, secondary to the onset of spasticity involving the intercostal and anterior abdominal muscles. In a related study, Ledsome and Sharp studied flow-volume curves and showed similar results. ${ }^{14}$ Inspiratory function can also improve if specific exercises are directed to strengthening of the diaphragm and accessory muscles.

As mentioned above, diaphragmatic and respiratory improvements can be noted over time and patients may recover neurological ability after injury. This can lead to improvements in vital capacity and the ability to wean mechanical ventilation. Ditunno et al proposed potential mechanisms underlying motor recovery following spinal cord injury ${ }^{15}$ and these would apply to the diaphragmatic functional recovery seen in this case presentation. The mechanisms for motor recovery include neurapraxic recovery (usually within 6 weeks), muscle fiber hypertrophy (from 2-6 months), peripheral nerve sprouting (2-6 months) and axonal regeneration (beyond 12 months). It would appear that axonal regeneration was primarily responsible for diaphragmatic recovery following this patient's discharge at 8 months post injury and it is likely that recovery could have been noted at an earlier time period if adequate respiratory reassessment had been undertaken.

Limited information is available in the literature regarding the recovery of phrenic nerve function following high cervical spinal cord injury. Lieberman studied patients with high cervical SCI cord injury and demonstrated a pattern of phrenic nerve conduction that changed over time. ${ }^{16}$ Nerves may be responsive initially and then become unresponsive, only to have responses reappear at a later date. $\mathrm{He}$ found that those phrenic nerves which were initially responsive, and later unresponsive, changed their function within the first 4 weeks post-injury. The phrenic nerves which regained function from a nonresponsive state did so within the first 270 days post injury. He concluded that phrenic nerve conduction studies should be carried out for at least 6 weeks post-injury on non-responsive nerves or those demonstrating a change in function before a final decision is made regarding phrenic nerve viability.

Initial assessment of the respiratory system following cervical spinal cord injury should include determination of diaphragmatic function. Physical examination can evaluate chest or abdominal wall expansion during inspiration. Fluoroscopy can assess appropriate spontaneous movement of the diaphragm and phrenic nerve conduction studies can document the viability of the phrenic nerve. Pulmonary function studies measure lung vital capacity and should be recorded on admission and then intermittently as necessary.

Re-evaluation of the need for mechanical ventilation following high cervical SCI is important secondary to increased patient morbidity and cost, along with diminished quality of life associated with ventilator-dependency. ${ }^{4.5}$ Respiratory complications remain a significant cause of morbidity and are the leading cause of death during the acute management of traumatic tetraplegia. ${ }^{3,13,19}$ Whiteneck reported a high rate of pulmonary complications and rehospitalization in chronic ventilator dependent tetraplegic patients. ${ }^{18}$ The loss of abdominal, intercostal and diaphragm muscles in progressively higher SCI results in a greater incidence of pulmonary problems in those with tetraplegic than those with paraplegia. ${ }^{19,20}$ Thus an aggressive pulmonary treatment program including bronchodilators, chest physical therapy, positioning, quad coughing and inspirometry is necessary.

Vigorous efforts should be undertaken to wean patients from mechanical ventilation. Literature reports that $60 \%$ of initially ventilatory dependent tetraplegic patients were eventually weaned and the 5 year survival was $84 \%$ in ventilatory independent tetraplegic patients as compared to $33 \%$ of ventilatory patients. ${ }^{4}$ As pulmonary functions and breathing mechanisms improve, weaning from mechanical ventilation can begin, and two methods are commonly utilized: Intermittent mandatory ventilation (IMV) and T-tube weaning. ${ }^{21,22}$ IMV gradually reduces the ventilatory rate while allowing patients to take spontaneous breaths in between the mechanical breaths. T-tube or progressive ventilatory-free breathing gradually increases ventilatory independent time periods, thus offering a training effect to the respiratory musculature. In a recent study by Peterson, ${ }^{21}$ T-tube weaning was found to be successful more often than IMV $(67 \%$ compared to $35 \%$ ) and appeared to be a more effective means of weaning high level tetraplegic patients. With increased survival of those with high tetraplegia there has become a greater need for criteria for the determination of successful ventilatory weaning and for methods of weaning patients from mechanical ventilation. Factors shown to adversely affect weaning are a vital capacity less than 1000 cc's, age $>50$ and the presence of associated injuries. ${ }^{4}$ Strengthening exercises and endurance training following cervical spinal cord injury is advantagious. Daily resistant inspiratory and expiratory muscle training has been noted to increase inspiratory pressure, prolong endurance and protect against fatigue. ${ }^{23,24}$ This method of training has been found to be beneficial in weaning high level tetraplegic patients from mechanical ventilation. ${ }^{25,26}$ 


\section{Conclusion}

The importance of re-evaluation of respiratory function and in particular, phrenic nerve viability and diaphragm function, in high cervical tetraplegic patients is stressed in this clinical report. Clinicians must consider the possible temporal changes in phrenic nerve and diaphragm function following acute SCI. Re-evaluation, utilizing physical examination, diaphragmatic fluoroscopy, phrenic nerve conduction studies, and pulmonary function tests, are required to appropriately assess respiratory status and, if possible, ventilatory weaning should be considered along with active respiratory exercise training.

\section{References}

1 Dureene J, Maclem P and Roussos C. The respiratory muscles: mechanism, control and pathophysiology. Am Rev Respir Dis 1978; 118: $119-133$.

2 Bergofsky E. Mechanism for respiratory insufficiency after cervical cord injury: a source of alveolar hypoventilation. Ann Intern Med 1964; 61: 435 - 447.

3 Carter R. Medical management of pulmonary complications of spinal cord injury. Adv Neurol 1979; 22: $261-269$.

4 Wicks $A$ and Menter R. Long-term outlook in quadriplegic patients with initial ventilator dependency. Chest 1986; 90: 406 410.

5 Goldberg A. Home care for a better life for ventilator-dependent people. Chest 1983; 84: $365-366$.

6 Donovan W and Taylor N. Ventilatory assistance in quadriplegia. Arch Phys Med Rehab 1973; 54: 485-488.

7 Ditunno J, Stover S and Freed M. Motor recovery of the upper extremities in traumatic quadriplegia: A multicenter study. Arch Phys Med Rehabil 1992; 73: 431 - 436.

8 DeTroyer A, Estenne $\mathbf{M}$ and Vincken W. Rib cage motion and muscle use in high tetraplegics. Am Rev Respir Dis 1986; 133: $1115-1119$.

9 Bach J and Alba A. Noninvasive options for ventilatory support of the traumatic high level quadriplegia. Chest 1990; 98: 613619.

10 Glenn W, Holcomb W and Shaw R. Long-term ventilatory support by diaphragm pacing in quadriplegia. Ann Surg 1976; 183: $566-577$.
11 Montero J, Feldman D and Montero D. Effects of glossopharyngeal breathing on respiratory function after cervical cord transection. Arch Phys Med Rehabil 1967; 48: 650-653.

12 Stone $D$ and Keltz $H$. The effect of respiratory muscle dysfunction on pulmonary function: studies in patients with spinal cord injuries. Amer Rev Resp Dis 1963; 88: 621 - 629.

13 McMichan J, Michel L and Westbrook P. Pulmonary dysfunction following traumatic quadriplegia. JAMA 1980; 243: 528 531 .

14 Ledsome $\mathbf{J}$ and Sharp J. Pulmonary function in acute cervical cord injury. Am Rev Respir Dis 1981; 124: $41-45$.

15 Ditunno J, Sipski M, Posuniak E and Chen Y. Wrist extensor recovery in traumatic quadriplegia. Arch Phys Med Rehab 1987; 68: $287-290$.

16 Lieberman J, et al. Serial phrenic nerve. Nerve conduction studies in candidates for diaphragmatic pacing. Arch Phys Med Rehab 1980; 61: $528-531$.

17 Bellany R, Pitts F and Stauffer E. Respiratory complications in traumatic quadriplegia; analysis of 20 years' experience. $J$ Neurosurg 1973; 39: 596-600.

18 Whiteneck G, Carter R and Hall K. Collaborative study of high quadriplegia. Arch Phys Med Rehabil 1985; 66: 575.

19 McKinley A, Auchincloss J and Gilbert R. Pulmonary function, ventilatory control and respiratory complications in quadriplegic subjects. Am Rev Resp Dis 1969; 100: 526532.

20 Forner J. Lung volumes and mechanics of breathing in tetraplegics. Paraplegia 1980; 18: $258-266$.

21 Peterson W, Charlifue $M$ and Gerhart R. Two methods of weaning persons with quadriplegia from mechanical ventilators. Paraplegia 1994; 32: 98 - 103.

22 Knebel A. Weaning from mechanical ventilation: current controversies. Heart Lung 1991; 20: 321 - 331 .

23 Gross D, Ladd $\mathrm{H}$ and Riley $\mathrm{E}$. The effect of training on strength and endurance of the diaphragm in quadriplegia. $A m \mathrm{~J} \mathrm{Med}$ 1980; 68: $27-35$

24 Huldtgren A, Fugl-Meyer A and Jonasson E. Ventilatory dysfunction and respiratory rehabilitation in post-traumatic quadriplegia. Eur J Respir Dis 1980; 61: 347 - 356.

25 Lerman R and Weiss M. Progressive resistive exercise in weaning high quadriplegics from the ventilator. Paraplegia 1987; 25: $130-135$.

26 Aldrich $\mathrm{T}$, Karpel $\mathbf{J}$ and Uhrlass R. Weaning from mechanical ventilation: adjunctive use of inspiratory muscle resistive training. Crit Care Med 1989; 17: 143-147. 